PREPARED FOR THE U.S. DEPARTMENT OF ENERGY, UNDER CONTRACT DE-AC02-76CH03073

PPPL-3861

PPPL-3861

UC-70

Gyrokinetic Simulations of Microinstabilities in Stellarator Geometry

by

J.L.V. Lewandowski

August 2003

N/M

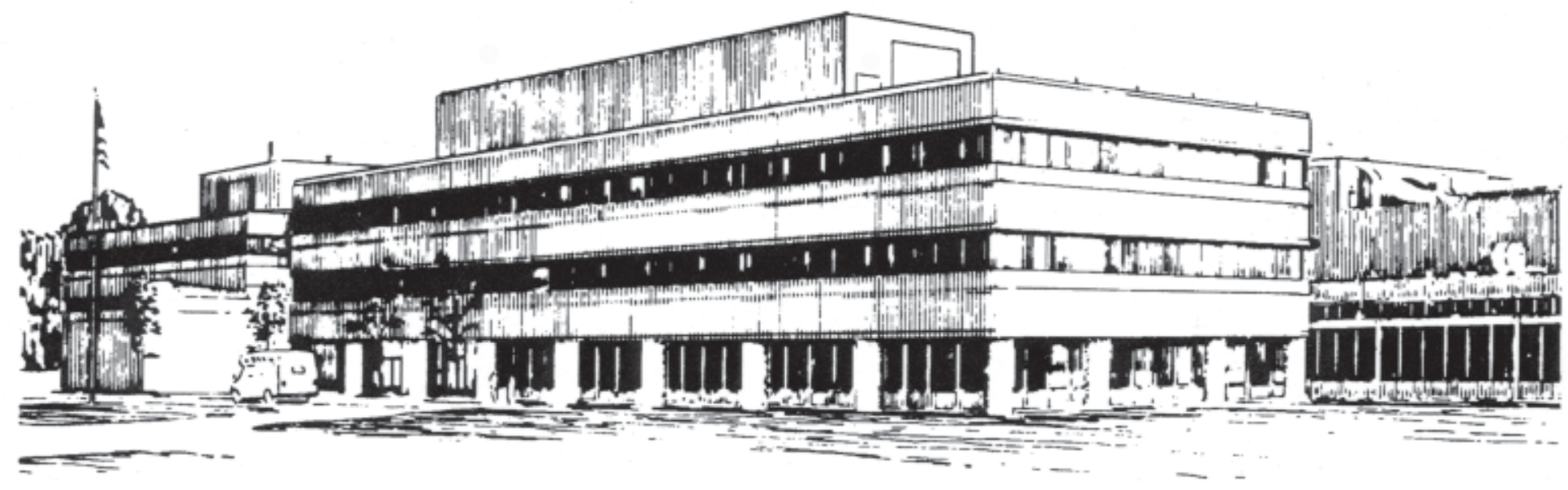

PRINCETON PLASMA PHYSICS LABORATORY PRINCETON UNIVERSITY, PRINCETON, NEW JERSEY 


\section{PPPL Reports Disclaimer}

This report was prepared as an account of work sponsored by an agency of the United States Government. Neither the United States Government nor any agency thereof, nor any of their employees, makes any warranty, express or implied, or assumes any legal liability or responsibility for the accuracy, completeness, or usefulness of any information, apparatus, product, or process disclosed, or represents that its use would not infringe privately owned rights. Reference herein to any specific commercial product, process, or service by trade name, trademark, manufacturer, or otherwise, does not necessarily constitute or imply its endorsement, recommendation, or favoring by the United States Government or any agency thereof. The views and opinions of authors expressed herein do not necessarily state or reflect those of the United States Government or any agency thereof.

\section{Availability}

This report is posted on the U.S. Department of Energy's Princeton Plasma Physics Laboratory Publications and Reports web site in Fiscal Year 2003. The home page for PPPL Reports and Publications is: http://www.pppl.gov/pub_report/

DOE and DOE Contractors can obtain copies of this report from:

U.S. Department of Energy

Office of Scientific and Technical Information

DOE Technical Information Services (DTIS)

P.O. Box 62

Oak Ridge, TN 37831

Telephone: (865) 576-8401

Fax: (865) 576-5728

Email: reports@adonis.osti.gov

This report is available to the general public from:

National Technical Information Service

U.S. Department of Commerce

5285 Port Royal Road

Springfield, VA 22161

Telephone: $1-800-553-6847$ or

(703) $605-6000$

Fax: (703) 321-8547

Internet: http://www.ntis.gov/ordering.htm 


\title{
Gyrokinetic simulations of microinstabilities in stellarator geometry
}

\author{
J.L.V. Lewandowski* \\ Princeton Plasma Physics Laboratory \\ Princeton University, P.O. Box 451 \\ Princeton NJ 08543 \\ USA
}

(Dated: August 25, 2003)

\begin{abstract}
A computational study of microinstabilities in general geometry is presented. The ion gyrokinetic is solved as an initial value problem. The advantage of this approach is the accurate treatment of some important kinetic effects. The magnetohydrodynamic equilibrium is obtained from a threedimensional local equilibrium model. The use of a local magnetohydrodynamic equilibrium model allows for a computationally-efficient systematic study of the impact of the magnetic structure on microinstabilities.
\end{abstract}

*Electronic address: jlewando@pppl.gov 


\section{INTRODUCTION}

It is now generally accepted in the fusion community that even if fast, large-scale magnetohydrodynamic (MHD) instabilities can be suppressed, magnetically-confined plasmas always contain sufficient free energy to drive slow, short-scale instabilities. These slow, short-scale instabilities, often called microinstabilities [1], are a major concern as confinement is concerned. The cross-field (perpendicular) transport associated with microinstabilities is often one to two orders magnitude larger than the neoclassical transport, and it is called 'anomalous' for this reason $[2,3]$.

Substantial efforts have been made to understand microinstability dynamics and the associated transport in tokamak geometry. However very little work has been published on microinstabilities in stellarator geometry. The earlier work of Bhattacharjee et al. [4] considered an electron drift wave model (with cold ions) for helically symmetric configurations; the main conclusion of the paper by Bhattacharjee et al. is that localized and extended modes can coexist in such configurations [4]. The spectrum of the model used by Bhattacharjee et al. has been calculated by Persson et al. [5]. The first drift wave calculations in realistic stellarator geometry were carried out by Dominguez and co-workers [7] for the dissipative trapped electron mode (DTEM); they showed that extended modes as well as strongly localized modes do exist (in the linear approximation) in a fully three-dimensional configuration.

As mentioned above, although the topic of drift wave stability and dynamics in tokamak plasma has been theoretically and numerically studied quite extensively, the study of low-frequency drift-type modes in stellarator geometry has received much less attention. One major reason for this state of affairs is that stellarator plasmas are inherently threedimensional and usually require the use of sophisticated equilibrium codes to specify their magnetohydrodynamic (MHD) equilibria. The most stringent linear stability considerations are usually based on the eikonal representation for perturbations; the problem is then reduced to an initial-value (or eigenvalue) problem along the magnetic field line; in general, the linear stability properties are studied one magnetic surface at a time. This observation is one motivation behind the three-dimensional local magnetohydrodynamic of Hegna [20]. Hegna's equilibrium model is particularly efficient for drift wave calculations as the MHD equilibrium is determined one surface at a time; this low-cost method allows us to study the 
effect of magnetic surface shaping (or its so-called parameterization) on drift wave stability. Although the initial parameterization of the magnetic surface in the local MHD equilibrium model can be quite general [see Eq.(9)], the main focus of this paper is a stellarator with an helical magnetic axis.

This paper is organized as follows; in section II, the basic physical model based on the ion gyrokinetic equation is presented in section II. The characterization of the local MHD equilibrium is given in section III. Section IV describes the numerical method used to solve the ion gyrokinetic equation as an initial-value problem. Numerical results are presented in section $\mathrm{V}$, and conclusions are given in section VI.

\section{ION GYROKINETIC MODEL}

The Ion-Temperature-Gradient-Driven (ITG) mode is a drift-type instability which arises from the free energy stored in the ion pressure gradient. In slab geometry and for a flat density profile, the mode is attributed to the coupling between the electron drift wave and the ion acoustic wave. Rudakov and Sagdeev considered a simple ITG model [9] in slab geometry and for a flat density profile, neglecting kinetic effects. The inclusion of magnetic shear and kinetic effects were investigated by Pogutse [10]. Using a fluid model and assuming an adiabatic response for the electrons, a systematic study of the mode structure of the ITG mode in sheared slab geometry was carried out by Coppi et al. [11], and by Hassam et al. $[12]$.

As shown by Horton et al. [13], the ITG mode in toroidal geometry is mainly driven by unfavorable magnetic curvature rather than the coupling of the electron drift wave to the ion acoustic wave. It is worth noting that other pressure-gradient-driven modes, such as the collisionless trapped-particle mode [14], can be driven unstable in the presence of unfavorable magnetic curvature.

The most unstable modes are strongly elongated along the direction of the equilibrium magnetic field $\left(k_{\|} / k_{\perp} \ll 1\right)$ and it is convenient to use the eikonal representation for each fluctuating quantity $\widetilde{f}$

$$
\widetilde{f}=\widehat{f}(\bar{\theta}, t) \exp (i N \alpha)
$$

where $N \gg 1$ is the toroidal mode number, $\alpha=\theta-\iota \zeta$ is the field line label, $\theta$ is the 
magnetic poloidal angle, $\zeta$ is the magnetic toroidal angle and $\iota$ is the rotational transform. Here $\widehat{f}(\bar{\theta}, t)$ is the amplitude which varies slowly with the extended poloidal angle, $\bar{\theta}$.

The ion distribution function can be written as $F=F_{0}+\delta f$, where $F_{0}$ and $\delta f$ are the equilibrium and perturbed parts of $F$, respectively. The equilibrium distribution for the ions must be independent of the gyro-angle and constant along the magnetic field line [8, 15]; these requirements are fulfilled for a Maxwellian distribution

$$
F_{M}=\frac{n_{0}}{\pi^{3 / 2} v_{\mathrm{thi}^{3}}} \exp \left(-\frac{v^{2}}{v_{\mathrm{thi}}{ }^{2}}\right)
$$

where $v_{\text {thi }}=\sqrt{2 T_{i}(\rho) / m_{i}}$ is the ion thermal velocity, $T_{i}$ is the equilibrium ion temperature which is assumed to be a function of the radial coordinate $\rho$ (which satisfies $\mathbf{B} \cdot \boldsymbol{\nabla} \rho \equiv 0$; see next section). The perturbed ion distribution function, $\delta f$, can be written as

$$
\delta f=\underbrace{-\frac{F_{M}}{\tau} \widetilde{\Phi}}_{\mathbf{1}}+\underbrace{h J_{0}}_{\mathbf{2}}+\underbrace{\frac{J_{0}^{2} F_{M}}{\tau} \widetilde{\Phi}}_{\mathbf{3}},
$$

where terms $\mathbf{1}$ and $\mathbf{2}$ are the adiabatic part and nondiabatic part of $\delta f$, respectively; term 3 arises becauses the guiding center density and the particle density do not coincide due to the finite ion Larmor radius and is called the polarization term for this reason. The nonadiabatic response $h$ satisfies the linear, collisionless gyro-kinetic equation [8]

$$
\frac{\partial h}{\partial t}=-v_{\|} \nabla_{\|}\left(h+\frac{\widetilde{\Phi}}{\tau} J_{0} F_{M}\right)-i \omega_{\mathrm{di}} h+i\left(\omega_{\star \mathrm{i}}-\omega_{\mathrm{di}}\right) \frac{\widetilde{\Phi}}{\tau} J_{0} F_{M}
$$

In Eqs $(3,4), \tau \equiv T_{i} / T_{e}, \widetilde{\Phi}=e \Phi / T_{e}, J_{0}$ is the zeroth-order Bessel function (arising from the averaging over the fast gyro-motion) with argument $\xi \equiv k_{\perp} v_{\perp} / \omega_{\mathrm{ci}}, \omega_{\mathrm{ci}}=e B /\left(m_{i} c\right)$ is the ion cyclotron frequency and $k_{\perp}$ is the magnitude of the perpendicular vector. Note that $\xi$ depends on the perpendicular velocity as well as the position along the field line through the spatial dependence of $B$ and $k_{\perp} \propto|\nabla \alpha|$. The effect of the global magnetic shear manifests itself in the secular behavior of $k_{\perp}$. In Eq. (4)

$$
\omega_{\star \mathrm{i}} \equiv \frac{c T_{i}}{e B}\left(\mathbf{k}_{\perp} \times \widehat{\mathbf{b}}\right) \cdot \frac{\nabla F_{M}}{F_{M}}
$$

is the velocity-dependent ion diamagnetic drift frequency and

$$
\omega_{\mathrm{di}} \equiv \omega_{\mathrm{ci}}^{-1}\left[\widehat{\mathbf{b}} \times\left(v_{\|}^{2} \boldsymbol{\kappa}+\frac{v_{\perp}^{2}}{2} \frac{\boldsymbol{\nabla} B}{B}\right)\right] \cdot \mathbf{k}_{\perp},
$$


is the velocity-dependent ion curvature drift frequency, $\widehat{\mathbf{b}} \equiv \mathbf{B} / B$ is the unit vector in the direction of the confining magnetic field, $\boldsymbol{\kappa} \equiv(\widehat{\mathbf{b}} \cdot \boldsymbol{\nabla}) \widehat{\mathbf{b}}$ is the magnetic curvature and $\left.\nabla_{\|} \equiv \nabla\right|_{\alpha}$ is the parallel gradient operator along the magnetic field line (i.e. keeping the field line label, $\alpha$, constant). The electrostatic potential is determined from the quasineutrality condition, which is valid for low-frequency modes [2],

$$
\int F_{i} d^{3} v=\int F_{e} d^{3} v
$$

Assuming that the electrons respond adiabatically to the electrostatic potential, and using Eq.(3), the quasineutrality condition takes the form of

$$
\widetilde{\Phi}=\frac{1}{n_{0} G(b)} \int J_{0} h d^{3} v
$$

where $G(b) \equiv 1+[1-\Gamma(b)] / \tau, b=k_{\perp}{ }^{2} \rho_{\text {thi }}{ }^{2} / 2, \Gamma(b) \equiv I_{0}(b) \exp (-b), I_{0}$ is the modified Bessel of order zero and the relation $[16]$

$$
\int_{0}^{\infty} \exp (-\alpha x) J_{\lambda}(2 \beta \sqrt{x}) J_{\lambda}(2 \gamma \sqrt{x}) d x=\frac{1}{\alpha} I_{\lambda}(2 \beta \gamma / \alpha) \exp \left(-\frac{\beta^{2}+\gamma^{2}}{\alpha}\right)
$$

has been used. The numerical solution of Eq.(4) as an initial value problem along the field line, coupled with the quasineutrality condition [Eq.(7)], is described in section IV. Note that Eqs. $(4,7)$ are quite general and can be applied in particular to the study of ITG stability in stellarator plasmas. In the next section, the MHD equilibrium, based on the original three-dimensional model of Hegna [20], is described.

\section{LOCAL MAGNETOHYDRODYNAMIC EQUILIBRIUM}

The confining magnetic field is written in straight-field line coordinates as

$$
\mathbf{B}=\nabla \psi \times \nabla \alpha
$$

where $\alpha \equiv \theta-\iota \zeta$ is the field line label, $\theta$ is the magnetic poloidal angle, $\zeta$ is the magnetic toroidal angle, $\iota$ is the rotational transform, and $\psi$ is related to the magnetic toroidal flux. Without loss of generality, one can specify the shape of the magnetic surface in terms of the 
cylindrical coordinates $(R, \phi, Z)$ as

$$
\begin{aligned}
R & =\sum_{m=0}^{M} \sum_{n=-N}^{N} R_{m n} \cos \left(\varphi_{m n}\right) \\
\phi & =-\zeta+\sum_{m=0}^{M} \sum_{n=-N}^{N} \phi_{m n} \sin \left(\varphi_{m n}\right) \\
Z & =\sum_{m=0}^{M} \sum_{n=-N}^{N} Z_{m n} \sin \left(\varphi_{m n}\right)
\end{aligned}
$$

where $\varphi_{m n} \equiv m \theta+n N_{p} \zeta, N_{p}$ is the number of field periods, and the poloidal and toroidal Fourier mode numbers $M$ and $N$, as well as the amplitudes $\left\{R_{m n}, \phi_{m n}, Z_{m n}\right\}$, are free parameters. Using the parameterization (9) one can determine the covariant basis vectors $\mathbf{e}_{\theta}=\partial \mathbf{r} / \partial \theta$ and $\mathbf{e}_{\zeta}=\partial \mathbf{r} / \partial \zeta$, where $\mathbf{r}$ is the position vector, and the metric elements $g_{\theta \theta}=\mathbf{e}_{\theta} \cdot \mathbf{e}_{\theta}, g_{\theta \zeta}=\mathbf{e}_{\theta} \cdot \mathbf{e}_{\zeta}$ and $g_{\zeta \zeta}=\mathbf{e}_{\zeta} \cdot \mathbf{e}_{\zeta}$. It is convenient to define an orthonormal vector set $\{\widehat{\mathbf{b}}, \widehat{\mathbf{n}}, \widehat{\mathbf{g}}\}$ attached to the magnetic field lines

$$
\begin{array}{rlrl}
\widehat{\mathbf{b}} & \equiv \frac{\mathbf{B}}{B}=\frac{\mathbf{e}_{\eta}}{\left|\mathbf{e}_{\eta}\right|} & & \text { (parallel) } \\
\widehat{\mathbf{n}} & \equiv \frac{\mathbf{e}_{\theta} \times \mathbf{e}_{\zeta}}{\left|\mathbf{e}_{\theta} \times \mathbf{e}_{\zeta}\right|} & & \text { (normal) } \\
\widehat{\mathbf{g}} & \equiv \widehat{\mathbf{b}} \times \widehat{\mathbf{n}} & \text { (geodesic) }
\end{array}
$$

where $\mathbf{e}_{\eta} \equiv \mathbf{e}_{\zeta}+\iota \mathbf{e}_{\theta}$. The vector set $\{\widehat{\mathbf{b}}, \widehat{\mathbf{n}}, \widehat{\mathbf{g}}\}$ can be determined from the parameterization (9). Finally, one can calculate important geometrical attributes of the confining $\mathbf{B}$ field such as the geodesic curvature, $\kappa_{g}=\widehat{\mathbf{g}} \cdot[(\widehat{\mathbf{b}} \cdot \nabla) \widehat{\mathbf{b}}]$, the normal curvature, $\kappa_{n}=\widehat{\mathbf{n}} \cdot[(\widehat{\mathbf{b}} \cdot \nabla) \widehat{\mathbf{b}}]$, and the normal torsion $\tau_{n}=-\widehat{\mathbf{n}} \cdot[(\widehat{\mathbf{b}} \cdot \nabla) \widehat{\mathbf{g}}]$. In order to determine the Jacobian of the transformation, $\mathcal{J}=[\boldsymbol{\nabla} \psi \cdot(\boldsymbol{\nabla} \theta \times \boldsymbol{\nabla} \zeta)]^{-1}=\mathbf{e}_{\psi} \cdot\left(\mathbf{e}_{\theta} \times \mathbf{e}_{\zeta}\right)$, we demand that the normal current vanishes everywhere on the magnetic surface [20]; using Ampere's law, one then has $J_{n} \equiv$ $\widehat{\mathbf{n}} \cdot \mathbf{J} \propto \boldsymbol{\nabla} \psi \cdot(\boldsymbol{\nabla} \times \mathbf{B}) \equiv 0$, or

$$
\frac{\partial}{\partial \theta}\left(\frac{F(\theta, \zeta)}{\partial}\right)=\frac{\partial}{\partial \zeta}\left(\frac{G(\theta, \zeta)}{\partial}\right),
$$

where

$$
\begin{aligned}
& F(\theta, \zeta)=g_{\zeta \zeta}+\iota g_{\theta \zeta} \\
& G(\theta, \zeta)=g_{\theta \zeta}+\iota g_{\theta \theta} .
\end{aligned}
$$


Eq.(11) introduces the primary constraint on the choice $\mathbf{r}=\mathbf{r}(\theta, \zeta)$ [Eq.(9)]. Eq.(11) is termed the Jacobian constraint. Note that in the axisymmetric case, the Jacobian constraint admits the exact solution of

$$
\mathcal{J}=f(\psi) F(\theta, \zeta)
$$

where $f(\psi)$ is an arbitrary flux surface quantity. In the general case, Eq.(11) must be solved numerically. Assuming that a solution has been found, one can construct $\boldsymbol{\nabla} \psi=\left(\mathbf{e}_{\theta} \times \mathbf{e}_{\zeta}\right) / \mathcal{J}$ and $\mathbf{B}=\mathbf{e}_{\eta} / \mathcal{J}$. The next step consists in calcultating the parallel current density consistent with the radial force balance equation and the quasineutrality condition. Using the radial force balance equation

$$
\mathbf{J} \times \mathbf{B}=c \boldsymbol{\nabla} p
$$

in the quasineutrality condition, $\boldsymbol{\nabla} \cdot \mathbf{J}=0$, one obtains

$$
\boldsymbol{\nabla} \cdot \mathbf{J}_{\|}=-\boldsymbol{\nabla} \cdot \mathbf{J}_{\perp}=2 c \frac{d p}{d \psi} \frac{|\nabla \psi|}{B} \kappa_{g},
$$

where the Jacobian constraint, Eq.(11), has been used. Substituting $\sigma \equiv \mathbf{J} \cdot \mathbf{B} / B^{2}=\langle\sigma\rangle+\widehat{\sigma}$ in Eq.(14), one arrives at the equation of

$$
\mathbf{B} \cdot \nabla \lambda=2 \frac{|\nabla \psi|}{B} \kappa_{g} .
$$

where $\lambda \equiv \widehat{\sigma} /\left(c p^{\prime}\right)$ (a prime denotes a derivative with respect to the toroidal flux function) and the quantity $|\nabla \psi| / B$ can be calculated directly from the parameterization (9):

$$
\frac{|\nabla \psi|}{B}=\sqrt{\frac{g_{\theta \theta} g_{\zeta \zeta}-g_{\theta \zeta}{ }^{2}}{g_{\zeta \zeta}+2 \iota g_{\theta \zeta}+\iota^{2} g_{\theta \theta}}} .
$$

The flux surface quantity $\langle\sigma\rangle$ is yet to be determined. The flux surface average of any function $F$ is defined as

$$
\langle F\rangle \equiv \frac{\int_{0}^{2 \pi} d \zeta \int_{0}^{2 \pi} d \theta \mathcal{J} F}{\int_{0}^{2 \pi} d \zeta \int_{0}^{2 \pi} d \theta \mathcal{J}}=\frac{1}{V^{\prime}} \int_{0}^{2 \pi} d \zeta \int_{0}^{2 \pi} d \theta \mathcal{J} F
$$

where $V$ is the plasma volume enclosed within $\psi$. Note that if $\mathcal{J}$ is a solution of Eq.(11), then $f(\psi) \mathcal{J}$ is also a solution of the same equation. Since the arbitrary flux surface function $f(\psi)$ is not known, the quantity $V^{\prime}$ is left undetermined; in practice, $V^{\prime}$ is used as an 
overall normalization factor. The flux surface quantity $\langle\sigma\rangle$ can be obtained through the local magnetic shear defined as $S \equiv \widehat{\mathbf{g}} \cdot \boldsymbol{\nabla} \times \widehat{\mathbf{g}}$, which can also be written as (Appendix A)

$$
S=\frac{|\nabla \psi|^{2}}{B^{2}} \mathbf{B} \cdot \boldsymbol{\nabla}\left(D+\zeta \frac{d \iota}{d \psi}\right)
$$

where

$$
D \equiv \frac{\iota \nabla \zeta \cdot \nabla \psi-\nabla \theta \cdot \nabla \psi}{\nabla \psi \cdot \nabla \psi}
$$

Using $\mathbf{B} \cdot \boldsymbol{\nabla} \zeta=1 / \mathcal{J}$ and noting that the flux surface average operator, $\langle\bullet\rangle$, annihilates the B. $\boldsymbol{\nabla}$ operator, we get from Eq.(18)

$$
\left\langle\frac{S B^{2}}{|\boldsymbol{\nabla} \psi|^{2}}\right\rangle=4 \pi^{2} \frac{\iota^{\prime}}{V^{\prime}} .
$$

Substituting $S=4 \pi J_{\|} /(c B)-2 \tau_{n}$ in the left-hand side of Eq.(20) and noting that $\langle\widehat{\sigma}\rangle \equiv 0$, one obtains

$$
\langle\sigma\rangle=c \sigma_{0}-c \sigma_{1} \frac{d p}{d \psi}-c \sigma_{2} \frac{d \iota}{d \psi},
$$

where $\sigma_{0}=C_{3} /\left(2 \pi C_{1}\right), \sigma_{1}=C_{2} / C_{1}$ and $\sigma_{2}=\pi / C_{1}$; we have defined

$$
\begin{aligned}
& C_{1}=\int_{0}^{2 \pi} d \zeta \int_{0}^{2 \pi} \frac{B^{2} \mathcal{\partial}}{g^{\psi \psi}} d \theta, \\
& C_{2}=\int_{0}^{2 \pi} d \zeta \int_{0}^{2 \pi} \frac{B^{2} \mathcal{\partial} \lambda}{g^{\psi \psi}} d \theta,
\end{aligned}
$$

and

$$
C_{3}=\int_{0}^{2 \pi} d \zeta \int_{0}^{2 \pi} \frac{B^{2} \partial \tau_{n}}{g^{\psi \psi}} d \theta
$$

In summary, given the parameterization (9), one solves Eq.(11) for the Jacobian, followed by Eq.(15) for $\lambda$ (which is proportional to the part of $J_{\|} / B$ that varies in the magnetic surface). Given $\iota^{\prime}$ and $p^{\prime}$ (free parameters) one calculates $\langle\sigma\rangle$ through Eq.(21) and the specification of the local MHD equilibrium is complete.

\section{NUMERICAL METHOD}

There is some freedom in defining the radial coordinate. In this paper, the radial coordinate is conveniently defined as $\rho \equiv R_{0} \sqrt{2 \bar{\psi}}$ where $\bar{\psi} \equiv \psi / \psi_{0}$ and $\psi_{0}=B_{0} R_{0}{ }^{2} ; R_{0} \equiv R_{00}$ 
is the major radius [in the large-aspect ratio tokamak equilibrium, $\psi \simeq B_{0} r^{2} / 2$ where $B_{0}$ is the magnetic field strength evaluated at the magnetic axis; it follows that $\rho=r$ in this case]. In the general 3D case, it is convenient to define a magnetic field strength of reference as $B_{0} \equiv\left\langle B^{2}\right\rangle^{1 / 2}$.

It is numerically convenient to solve for $\widehat{g} \equiv \widehat{h} / F_{M}$ [as before, $\widehat{h}(\bar{\theta}, t)$ denotes the amplitude of $\widetilde{h}$ in the eikonal representation, Eq.(1)] rather than $\widehat{h}$. Using the eikonal representation [Eq.(1)] in Eq.(4) one obtains

$$
\frac{\partial \widehat{g}}{\partial \bar{t}}=-\sqrt{2 \tau} \frac{\epsilon_{n}}{q} V_{\|} S_{\|} \frac{\partial}{\partial \bar{\theta}}(\widehat{g}+\widehat{\phi})-i \Omega_{\mathrm{di}} \widehat{g}+i\left(\Omega_{\star \mathrm{i}}-\Omega_{\mathrm{di}}\right) \widehat{\phi},
$$

where $\widehat{\phi} \equiv J_{0} \widehat{\Phi} / \tau, \bar{t} \equiv \omega_{\star} t$ is the normalized time, $\omega_{\star}=c_{s} / L_{n}$ is the drift frequency, $c_{s}=\sqrt{T_{e} / m_{i}}$ is the ion sound speed, $\Omega_{\star \mathrm{i}}=\omega_{\star \mathrm{i}} / \omega_{\star}, \Omega_{\mathrm{di}} \equiv \omega_{\mathrm{di}} / \omega_{\star}$ and $V_{\|}=v_{\|} / v_{\text {thi }}$ is the normalized parallel velocity and $\epsilon_{n} \equiv L_{n} / R_{0}$. Finally $S_{\|}(\bar{\theta})=1 /(\bar{g} \bar{B})$ where $\bar{\jmath}=\left[R_{0} \nabla \bar{\psi} \cdot\left(R_{0} \boldsymbol{\nabla} \theta \times R_{0} \boldsymbol{\nabla} \zeta\right)\right]^{-1}$ is the dimensionless Jacobian and $\bar{B}=B / B_{0}=\mathcal{O}(1)$. The normalized diamagnetic frequency and the normalized curvature drift frequency can be written as

$$
\Omega_{\star \mathrm{i}}=-\tau k_{\theta} \rho_{s}\left[1+\eta_{i}\left(V^{2}-\frac{3}{2}\right)\right]
$$

and

$$
\Omega_{\mathrm{di}}=2 \tau \epsilon \epsilon_{n} k_{\theta} \rho_{s} S_{\perp}(\bar{\theta})\left(V_{\|}^{2}+\frac{V_{\perp}^{2}}{2}\right)
$$

respectively. In the above equations, $\eta_{i}=L_{n} / L_{T_{i}}, k_{\theta} \equiv N_{0} / \bar{a}$ is the characteristic perpendicular wavevector (i.e. $|\nabla \alpha| \frac{2}{\bar{\theta}=0} \sim k_{\theta}^{2}$ ), $V_{\perp}=v_{\perp} / v_{\text {thi }}, \epsilon=\bar{a} / R_{0}$ is the inverse aspect ratio and $\bar{a}$ is the minor radius defined as

$$
\bar{a} \equiv \frac{1}{2 \pi} \int_{0}^{2 \pi}\left|\mathbf{r}-\mathbf{R}_{0}\right|_{\zeta=0} d \theta
$$

where $\mathbf{R}_{0} \equiv R_{0,0} \widehat{\mathbf{R}}$ is the major radius vector. Using the general parameterization of Eq.(9) one can show that (Figure 1)

$$
\left|\mathbf{r}-\mathbf{R}_{0}\right|_{\zeta=0}=\left[\left(\sum_{m=1}^{M} R_{m, 0} \cos (m \theta)\right)^{2}+\left(\sum_{m=1}^{M} Z_{m, 0} \sin (m \theta)\right)^{2}\right]^{1 / 2} .
$$

The geometrical quantity $S_{\perp}(\bar{\theta})$, which is related to the curvature drift term in the ballooning representation, is given by

$$
S_{\perp}(\bar{\theta}) \equiv \frac{\kappa_{n}+\Lambda \kappa_{g}}{\sqrt{g \overline{\psi \psi}}}
$$


where

$$
\Lambda(\bar{\theta}) \equiv-\frac{\boldsymbol{\nabla} \psi \cdot \nabla \alpha}{B}
$$

As mentioned in section II, the global shear manifests itself in the secular behavior of $k_{\perp} \propto|\nabla \alpha|$. In particular, the argument of the Bessel function that arises from the gyroaveraging procedure is $\xi=k_{\perp} v_{\perp} / \omega_{\text {ci }}$ or

$$
\xi=\sqrt{2 \tau}\left(k_{\theta} \rho_{s}\right) \epsilon \sqrt{\mathcal{L}} V_{\perp}
$$

with the definition of

$$
\mathcal{L}(\bar{\theta}) \equiv \frac{1+\Lambda^{2}}{R_{0}^{2} g^{\overline{\psi \psi}}}
$$

As mentioned above, the curvature contributions in Eq.(22) [terms proportional to $\Omega_{\mathrm{di}}$ ] display a secular behavior for large $\bar{\theta}$, which can be problematic from the numerical point of view. To highlight the difficulty associated with the curvature term, we write Eq.(22) as

$$
\frac{\partial \widehat{g}}{\partial \bar{t}}+i \Omega_{\mathrm{di}} \widehat{g}=Q
$$

where

$$
Q=-\sqrt{2 \tau} \frac{\epsilon_{n}}{q} V_{\|} S_{\|} \frac{\partial}{\partial \bar{\theta}}(\widehat{g}+\widehat{\phi})+i\left(\Omega_{\star \mathrm{i}}-\Omega_{\mathrm{di}}\right) \widehat{\phi}
$$

Let us denote $\widehat{g}^{(n)}$ the solution at time step $n$. The explicit algorithm associated with Eq.(29) is simply

$$
\frac{\widehat{g}^{(n+1)}-\widehat{g}^{(n)}}{\Delta \bar{t}}+i \Omega_{\mathrm{di}} \widehat{g}^{(n)}=Q^{(n)}
$$

where $\Delta \bar{t}$ denotes the time step. In order to address the numerical stability of scheme (30), let $\widehat{g} \sim \exp (\nu \bar{t})$. In the homogeneous case, $Q=0$, one arrives at $\widehat{g}^{(n+1)}=A \widehat{g}^{(n)}$ where the amplification factor is given by

$$
A=1-i \Omega_{\mathrm{di}} \Delta \bar{t}
$$

Clearly $|A|>1$ for any time step $\Delta \bar{t}>0$ and the explicit scheme (30) is unstable; note that the numerical instability is exacerbated because of the secular behavior of $\Omega_{\mathrm{di}}$ for large $\bar{\theta}$. Actually, the form of Eq.(29), together with the secular behavior of $\Omega_{\mathrm{di}}$, suggests the use 
of a semi-implicit scheme. In this scheme, the left-hand side of Eq.(29) is treated implicitly whereas its right-hand side is, as before, treated explicitly; this results in a discretized equation of the form

$$
\frac{\widehat{g}^{(n+1)}-\widehat{g}^{(n)}}{\Delta \bar{t}}+i \Omega_{\mathrm{di}} \widehat{g}^{(n+1)}=Q^{(n)},
$$

which admits the trivial solution of

$$
\widehat{g}^{(n+1)}=\frac{\widehat{g}^{(n)}+\Delta \bar{t} Q^{(n)}}{1+i \Omega_{\mathrm{di}} \Delta \bar{t}} .
$$

We note that the scheme (31) is unconditionally stable. In addition to the stability property of this scheme, it is also suppresses the 'noisy pattern' (due to the secular behavior of $\Omega_{\mathrm{di}}$ for $|\bar{\theta}| \gg 1$ ) that would arise if one would use an explicit scheme, such as the Lax-Wendroff scheme for example.

Since the ion gyro-kinetic equation is solved as an initial value problem the linear growth rate and the mode frequency must be computed dynamically. Another consequence associated with the initial value approach is that only the fastest growing mode is observed. Earlier studies of drift wave stability in realistic stellarator geometry [5, 21] have shown that the drift wave spectrum can have a rather complicated structure; in some case, it can be difficult to 'pick up' the most unstable mode. In order to determine the mode frequency, $\omega$, we use the transformation of

$$
\widehat{\Phi}(\bar{\theta}, t) \mapsto \bar{\Phi}(\bar{\theta}) \exp (-i \omega t)
$$

for the mode amplitude. The normal mode frequency can be written as $\omega=\Re(\omega)+i \Im(\omega) \equiv$ $\omega_{r}+i \gamma$. Noting that

$$
\frac{\partial \widehat{\Phi}}{\partial t}=\frac{1}{2} \frac{1}{|\widehat{\Phi}|} \frac{\partial}{\partial t}|\widehat{\Phi}|^{2}-i \omega_{r}|\widehat{\Phi}|
$$

we obtain

$$
\gamma=\frac{1}{\left|\langle\widehat{\Phi}\rangle_{\bar{\theta}}\right|} \frac{\partial}{\partial t}\left|\langle\widehat{\Phi}\rangle_{\bar{\theta}}\right|
$$

for the linear growth rate and

$$
\omega_{r}=-\Im\left(\frac{1}{\langle\widehat{\Phi}\rangle_{\bar{\theta}}} \frac{\partial}{\partial t}\langle\widehat{\Phi}\rangle_{\bar{\theta}}\right),
$$


for the mode frequency. In the above equations, we have defined the field-line average operator as

$$
\langle F\rangle_{\bar{\theta}} \equiv \frac{1}{2 \bar{\theta}_{\max }} \int_{-\bar{\theta}_{\max }}^{+\bar{\theta}_{\max }} F\left(\bar{\theta}^{\prime}, t\right) d \bar{\theta}^{\prime}
$$

for any function $F(\bar{\theta}, t)$. The free parameter in Eq.(34) must be large enough so that the linear growth rate [Eq.(32)] and the mode frequency [Eq.(33)] become independent of $\bar{\theta}_{\text {max }}$; a convergence study using this parameter is presented in the next section.

\section{NUMERICAL RESULTS}

As described in section III, the specification of the local MHD equilibrium depends on two free parameters, $\iota^{\prime}$ and $p^{\prime}$. It is convenient to use normalized quantities instead of $\iota^{\prime}$ and $p^{\prime}$; to make the connection with the standard large-aspect ratio tokamak case, we define the ballooning parameter

$$
\alpha_{b} \equiv \frac{2}{\pi^{2} \iota^{2}} \frac{V^{\prime}}{\left\langle\frac{B^{2}}{g^{\psi \psi}}\right\rangle^{1 / 2}}
$$

and the global shear parameter

$$
s_{b} \equiv-\frac{4 \pi^{2} R_{0}}{V^{\prime}} \frac{\frac{1}{\iota} \frac{d \iota}{d \psi}}{\left\langle\frac{B^{2}}{g^{\psi \psi}}\right\rangle} .
$$

Definitions $(35,36)$ can be related to the usual parameters $\alpha$ and $\widehat{s}$ of Connor et al. [19] for the case of a tokamak with circular, magnetic surfaces. For example, the global shear parameter can be related to the usual parameter $\widehat{s}$ as follows. The enclosed toroidal flux is $\psi_{T} \simeq \pi B_{0} r^{2}$, where $B_{0}$ is the magnetic field strength on the magnetic axis; accordingly, we have $\psi=\psi_{T} /(2 \pi) \simeq B_{0} r^{2} / 2$ for the toroidal flux function. Note that the plasma volume enclosed within $r$ is $V=2 \pi^{2} r^{2} R_{0}$ and that $g^{\psi \psi}=(d \psi / d r)^{2} \nabla r \cdot \nabla r$ it follows that

$$
s_{b}=\frac{4 \pi^{2} R_{0}}{\frac{d V}{d r}} \frac{\frac{1}{q} \frac{d q}{d r}}{\left\langle\frac{B^{2}}{g^{\psi \psi}}\right\rangle}=\frac{r}{q} \frac{d q}{d r} \equiv \widehat{s} .
$$

As mentioned in the Introduction, the initial parameterization of the magnetic surface [Eq.(9)] is quite general. However, in order to illustrate the usefulness and efficiency of 
the local equilibrium model for drift wave stability calculations, we consider the parameterization of

$$
\begin{aligned}
R & =R_{0}\left[1+\epsilon_{\mathrm{t}} \cos \theta+\epsilon_{\mathrm{h}} \cos \left(N_{p} \zeta\right)\right] \\
\phi & =-\zeta \\
Z & =R_{0}\left[\epsilon_{\mathrm{t}} \sin \theta+\epsilon_{\mathrm{h}} \sin \left(N_{p} \zeta\right)\right]
\end{aligned}
$$

where, as before, $N_{p}$ is the number of field periods; $\epsilon_{\mathrm{t}}$ and $\epsilon_{\mathrm{h}}$ are termed the toroidicity parameter and the helical parameter, respectively. In the remaining of this paper, we refer to Eq.(37) as the helical parameterization. Note that the case $\epsilon_{\mathrm{h}}=0$ in Eq.(37) corresponds to the tokamak parameterization with concentric, circular magnetic surfaces (which is valid for a low- $\beta$ plasma). When the parameter $\epsilon \equiv N_{p}^{2} \epsilon_{\mathrm{t}} \epsilon_{\mathrm{h}}$ is less than unity, the Jacobian for the helical parameterization can be calculated analytically (Appendix B)

$$
\mathcal{J}=\frac{X^{2}(\theta)}{A_{0}(\psi) g(\varphi)}
$$

where $\varphi \equiv N_{p} \zeta-\theta$ is the helical coordinate, $A_{0}(\psi)$ is an arbitrary flux function, $X(\theta)=$ $R_{0}\left(1+\epsilon_{\mathrm{t}} \cos \theta\right)$ and

$$
g(\varphi)=1+a(1-\cos \varphi)-b \sin ^{2} \varphi
$$

Here $a=\xi(1+\xi / \widehat{\alpha}) / \widehat{\alpha}, b=\xi(\widehat{\beta}+\xi) /\left(2 \widehat{\alpha}^{2}\right), \widehat{\alpha}=1+\epsilon_{\mathrm{t}}^{2}\left(N_{p} \iota+\frac{1}{2}\right)+\epsilon_{\mathrm{h}}{ }^{2}\left(N_{p}^{2}+\frac{1}{2}\right), \widehat{\beta}=$ $\widehat{\epsilon}\left[1+N_{p}\left(N_{p}+\iota\right)\right]$ and $\xi=\left[N_{p}\left(N_{p}+\iota\right)-1\right] \epsilon_{\mathrm{t}} \epsilon_{\mathrm{h}}$. Note that for the case of the tokamak parameterization $\left(\epsilon_{\mathrm{h}}=0\right)$ we have $a=b=\xi=0$ so that $g(\varphi)=1$ and Eq.(38) then reduces to the exact solution of

$$
\mathcal{J}=C\left(1+\epsilon_{\mathrm{t}} \cos \theta\right)^{2}
$$

where $C$ is an arbitrary multiplicative constant. When the parameter $\epsilon$ is comparable to unity, one must resort to the numerical solution described at the end of section III.

In all simulations reported in this paper, the initial profile for $\widehat{g}$ has been chosen to be a Gaussian profile with its maxima centered at $\bar{\theta}=0$

$$
\widehat{g}(t=0)=\widehat{g}_{0} \exp \left(-\sigma \bar{\theta}^{2}\right)
$$

where $\widehat{g}_{0}$ is a small amplitude and $\sigma>0$. The initial profile for the electrostatic potential can then be determined through the quasineutrality condition [Eq.(7)]; the system of equations 
is then advanced in time using the semi-implicit method described in the previous section. The distribution function is discretized on a three-dimensional grid $\widehat{g}\left(\bar{\theta}, V_{\|}, V_{\perp} ; t\right)$ whereas the electrostatic potential is discretized on a one-dimensional grid $\widehat{\Phi}(\bar{\theta} ; t)$. In order to benchmark our code, we have compared our results with the toroidal ITG eigenmode analysis of Guzdar et al. [18], which retains all the ion kinetic effects but assumes adiabatic electrons, as in the present paper. Gudzar's eigenmode analysis is valid for large-aspect-ratio, axisymmetric tokamak plasmas. Figure 2 shows the linear growth rate, normalized to $\omega_{\star}$, as a function of the temperature gradient parameter, $\eta_{i}$. The parameters are: $b=\left(k_{\theta} \rho_{s}\right)^{2}=0.1, q=\tau=$ $s_{b}=1.0, \epsilon_{n}=L_{n} / R_{0}=0.1$ and $\epsilon_{\mathrm{h}}=0$. The measured linear growth rate (plain line) is in good agreement with the numerical results based on the eigenmode analysis (dotted line) of Guzdar et al [18].

Figure 3 shows the time evolution of the linear growth rate (plain line) for a three-field period $\left(N_{p}=3\right)$ stellarator with an helical parameter of $\epsilon_{\mathrm{h}}=0.04$; other parameters are: $\epsilon_{\mathrm{t}}=0.1, k_{\theta} \rho_{\mathrm{s}}=0.8, q=2, \eta_{i}=2.0, s_{b}=0.9, \alpha_{b}=0.0, \epsilon_{n}=0.1$ and $\tau=T_{\mathrm{i}} / T_{\mathrm{e}}=1$. For comparison, the linear growth rate based on the time variation of $|\widehat{\Phi}|$ at $\bar{\theta}=0$

$$
\gamma=\frac{1}{|\widehat{\Phi}|_{\bar{\theta}=0}} \frac{\partial}{\partial t}|\widehat{\Phi}|_{\bar{\theta}=0},
$$

is shown as a dotted line. In the early stage of the simulation, the growth rates given by Eqs. $(32,40)$ do not agree indicating an internal adjustment between $\widehat{g}$ and $\widehat{\Phi}$ as their initial $\bar{\theta}$ dependence does not correspond to the most unstable eigenfunction of the system. Based on the asymptotic behavior of $\gamma / \omega_{\star}$ one can infer that the typical growth time of the instability is $\tau=1 / \gamma \approx 20 \omega_{\star}$, in good agreement with the time necessary for the transitory effects to disappear. Figure 4 shows the mode frequency (plain line) as a function of the normalized time $\omega_{\star} t$ for the same case as in Figure 3. The mode frequency based on

$$
\omega_{r}=-\Im\left(\frac{1}{\widehat{\Phi}_{\bar{\theta}=0}} \frac{\partial}{\partial t} \widehat{\Phi}_{\bar{\theta}=0}\right),
$$

is also shown (dotted line). Here again transitory effects disappear after a few growth times. The mode frequencies computed using two different methods [Eqs. $(33,41)]$ agree with each other for $t \gg \tau$.

The real (plain line) and imaginary (dotted line) parts of the electrostatic potential (most unstable eigenfunction) at the end of the simulations $\left(\omega_{\star} t=60\right)$ is shown in Figure 5 . Note 
that the mode 'balloons' on the outboard side of the torus (which corresponds to $\bar{\theta}=0$ ). The boundary conditions at large $\bar{\theta}$ are well satisfied. The eigenfunction associated with the most unstable mode is clearly square integrable, that is

$$
\int_{-\infty}^{+\infty}|\widehat{\Phi}|^{2} d \bar{\theta}<\infty
$$

As discussed in the previous section, the linear growth rate as computed by Eq.(32), depends implicitly on the value of $\bar{\theta}_{\max }$. Therefore, this parameter must be varied until $\partial \gamma / \partial \bar{\theta}_{\max } \simeq 0$. Figure 6 shows the saturated linear growth rate $(t / \tau \gg 1)$ as a function of the parameter $\bar{\theta}_{\max }$. The parameters are: $\epsilon_{\mathrm{t}}=0.1, \epsilon_{\mathrm{h}}=0.03, \eta_{i}=2, k_{\theta} \rho_{\mathrm{s}}=0.4, q=2$ and $\epsilon_{n}=L_{n} / R_{0}=0.1$. In Figure $6, \gamma_{\infty}$ denotes $\gamma$ for $\bar{\theta}_{\max } \mapsto \infty$. As it can be expected the linear growth rate (and the real part of the mode frequency) is strongly dependent on the value of $\bar{\theta}_{\max }$ when the value of this parameter is too small. For $\bar{\theta}_{\max }>5$ the linear growth rate becomes practically independent of the value of this parameter. Note that the parameter $\bar{\theta}_{\max }$ must be increased as the global shear parameter, $s_{b}$, is decreased. The same is true when the toroidal mode number, $N \propto k_{\theta} \rho_{\mathrm{s}}$, is decreased.

As discussed earlier in this section, the normalized distribution function $\widehat{g}$ is discretized on a finite velocity grid. The grid in velocity space is in the range of

$$
\begin{aligned}
& V_{\|} \in\left[-V_{\| \max },+V_{\| \max }\right], \\
& V_{\perp} \in\left[0, V_{\perp \max }\right],
\end{aligned}
$$

where $V_{\| \max }$ and $V_{\perp \max }$ are free parameters that must be varied to ensure proper convergence. Figure 7 shows the saturated linear growth rate as a function of $V_{\perp \max }$ (the parameter $V_{\| \max }$ is kept fixed at $\left.V_{\| \max } / v_{\text {thi }}=5\right)$. When the parameter is close to unity an important part of the density response, and therefore of the $\Phi$ response, is left out and the resulting linear growth rate is smaller than what it should be. A similar dependence of the linear growth rate on $V_{\| \max }$ is observed. All simulations reported in this paper were carried out with $V_{\| \max } / v_{\text {thi }}=V_{\perp \max } / v_{\text {thi }}=5$.

Figure 8 shows the dependence of the mode frequency as a function of the toroidal mode number parameter, $k_{\theta} \rho_{\mathrm{s}}$, for a three-field period stellarator. The parameters are: $\epsilon_{\mathrm{t}}=0.1$, $\epsilon_{\mathrm{h}}=0.04, \eta_{i}=2, q=2, s_{b}=0.9, \alpha_{b}=0, L_{n} / R_{0}=0.1$ and $\tau=1$. As expected, the mode rotates faster in the ion diamagnetic direction as the parameter $k_{\theta} \rho_{\mathrm{s}}$ is increased. Figure 9 shows the linear growth rate as a function of $k_{\theta} \rho_{\mathrm{s}}$ for the same parameters as in Fig. 8. The linear growth rate peaks at around $k_{\theta} \rho_{\mathrm{s}}=0.55$. 
One advantage of the local equilibrium model is that one can modify the MHD equilibrum dynamically for a very low computational cost. This is illustrated in Fig. 10 and Fig. 11 for a $N_{p}=3$ field period stellarator. In these figures, the helical parameter $\epsilon_{\mathrm{h}}$, which is related to the helical excursion of the magnetic axis, has been varied while the toroidicity parameter has been kept fixed at $\epsilon_{\mathrm{t}}=0.1$. The parameters are: $k_{\theta} \rho_{\mathrm{s}}=0.6, \eta_{i}=2.0$, $q=2, s_{b}=0.9, \alpha_{b}=0$ and $L_{n} / R_{0}=0.1$. As the parameter $\epsilon_{\mathrm{h}}$ is increased, the mode rotates faster in the ion diamagnetic direction as it can be seen in Fig.10. When the helical parameter, $\epsilon_{\mathrm{h}}$, is comparable to the toroidicity parameter, $\epsilon_{\mathrm{t}}$, key attributes of the magnetic configuration will be affected. In particular, the normal magnetic curvature, which drives the mode unstable in the outboard side of the torus, will be substantially modified. This is also true for the local magnetic shear and the geodesic magnetic curvature. Figure 11 shows the linear growth rate as a function of the helical parameter; all parameters are the same as in Fig. 10. The linear growth rate is almost independent of $\epsilon_{\mathrm{h}}$ when $\epsilon_{\mathrm{h}} \ll \epsilon_{\mathrm{t}}$; it is modified when $\epsilon_{\mathrm{h}} \sim \epsilon_{\mathrm{t}}$ (note that in this case the helical curvature dominates over the toroidal curvature). In order to understand the dependence of the linear growth rate on the helical parameter, one can compare the key attributes of the magnetic configuration for specific sets $\left(\epsilon_{\mathrm{t}}, \epsilon_{\mathrm{h}}\right)$; such key attributes are the normal and geodesic components of the magnetic curvature $\left(\kappa_{n}\right.$ and $\kappa_{g}$, respectively), the magnetic shear, $S$, and the normal torsion, $\tau_{n}$. Other quantities that characterize the equilibrium configuration do enter the drift wave stability calculations; however, the impact of these quantities are subdominant. The normal magnetic curvature and the magnetic shear are probably the most important quantities that affect drift wave (and ballooning) stability [21]. Figure 12 shows the normal curvature (plain line) and the local magnetic shear (dotted line) along the field line for the case of $\left(\epsilon_{\mathrm{t}}, \epsilon_{\mathrm{h}}\right)=(0.1,0.0)$. Figure 12 shows that the normal curvature is destabilizing (negative) in the outboard side of the torus. The global component (flux surface average part) of $S$ accounts for the bulk of the local magnetic shear. Figure 13 shows the normal magnetic curvature (plain line) and the local magnetic shear (dotted line) along the field line for the case of $\left(\epsilon_{\mathrm{t}}, \epsilon_{\mathrm{h}}\right)=(0.1,0.12)$. The variation of the local magnetic shear is larger than for the case $\left(\epsilon_{\mathrm{t}}, \epsilon_{\mathrm{h}}\right)=(0.1,0.0)$ because of the fast variation associated with the helical component. The same observation applies to the normal magnetic curvature. Fig. 12 and Fig. 13 show that $\kappa_{n}$ is more unfavorable when $\epsilon_{\mathrm{t}} \sim \epsilon_{\mathrm{h}}$. Furthermore the local magnetic shear becomes more positive around $\bar{\theta}=0$ (where the bulk of the mode is localized) as compared with 
the $\left(\epsilon_{\mathrm{t}}, \epsilon_{\mathrm{h}}\right)=(0.1,0.0)$ case. Therefore, both the normal curvature and the local magnetic shear favor a more unstable mode in the case of $\left(\epsilon_{\mathrm{t}}, \epsilon_{\mathrm{h}}\right)=(0.1,0.12)$ than for the case of $\left(\epsilon_{\mathrm{t}}, \epsilon_{\mathrm{h}}\right)=(0.1,0.0)$, in agreement with Fig.11. The detrimental influence of a large, positive local magnetic shear on drift wave stability in realistic 3D stellararator geometries has been noted by Nadeem and co-workers [22]; these authors also discuss the case of large, negative local magnetic shear which appears to have a stabilizing influence on the drift mode. This is in agreement with our observations, although the MHD model equilibrium used here is far simpler than the fully 3D stellarator equilibrium used in the work of Nadeem et al.

Finally, another attractive feature of the local MHD equilibrium model is that it allows for fast, computationally-efficient estimates of the anomalous diffusion coefficient, $D_{\perp}$; such estimates, however, have to be used with caution. Based on mixing length arguments [2], one can estimate the perpendicular diffusion coefficient using the relation of

$$
D_{\perp} \simeq D_{\perp}^{(\mathrm{m})} \equiv \frac{\gamma}{k_{r}^{2}}
$$

where $\gamma$ is the linear growth rate and $k_{r}$ is the magnitude of a typical radial wavevector. Using a general non-Markovian Fokker-Planck treatment, Zagorodny and Weiland [6] showed that memory effects can be important for the description of transport under saturared turbulence; they derived an estimate for the perpendicular diffusion coefficient of the form

$$
D_{\perp} \simeq D_{\perp}^{(\mathrm{fp})} \equiv \frac{\gamma^{3} / k_{r}^{2}}{\omega_{\mathrm{r}}^{2}+\gamma^{2}}=\frac{1}{1+\left(\omega_{\mathrm{r}} / \gamma\right)^{2}} D_{\perp}^{(\mathrm{m})} .
$$

We note that $D_{\perp}^{(\mathrm{fp})} \mapsto D_{\perp}^{(\mathrm{m})}$ when $\gamma \gg \omega_{\mathrm{r}}$. An estimate for $k_{r}$ in Eqs. $(43,44)$ can be obtained by a suitable average (weighted with the eigenfunction) of the normal component of $\boldsymbol{\nabla} \alpha \propto \mathbf{k}_{\perp}$

$$
k_{r}^{2}=\frac{\int_{-\infty}^{+\infty}\left(\mathbf{k}_{\perp} \cdot \widehat{\mathbf{n}}\right)^{2}|\widehat{\Phi}|^{2} d \bar{\theta}}{\int_{-\infty}^{+\infty}|\widehat{\Phi}|^{2} d \bar{\theta}}
$$

where

$$
\mathbf{k}_{\perp} \cdot \widehat{\mathbf{n}}=-\frac{N B}{\sqrt{g^{\psi \psi}}} \Lambda(\bar{\theta})
$$

Here $\Lambda(\bar{\theta})$, defined in Eq.(26), is related to the integrated local magnetic shear. Figure 14 shows the estimates for $D_{\perp}$ based on the mixing length theory (squares) and the nonMarkovian Fokker-Planck theory (triangles). The most striking feature is that $D_{\perp}^{(\mathrm{fp})}$ remains 
almost independent of the helical parameter for moderate $\epsilon_{\mathrm{h}}$; the mixing length-based diffusion coefficient, however, shows a clear upward trend with increasing $\epsilon_{\mathrm{h}}$. Therefore, although the linear growth rate does increase with an increase in the helical parameter, the non-Markovian Fokker-Planck diffusion coefficient does not predict a substantial decrease in the confinement time. More work is needed to resolve this issue especially when effects such as plasma shaping are taken into account.

\section{CONCLUSIONS}

Drift wave stability calculations in stellarator geometry usually requires the use of sophisticated, computationally-intensive 3D MHD equilibrium codes. However, as far as linear

stability is concerned, the general approach is to study the local drift wave stability using the ballooning representation, one magnetic surface at a time. Hegna's local equilibrium model [20] is specific to a particular magnetic surface and it is computationally very efficient.

The local equilibrium model has been used to study microinstabilities in a stellarator configuration with an helical axis. The stability analysis presented in this paper is based on the ion gyrokinetic equation, including all kinetic effects, with adiabatic electrons. Particle trapping has not been taken account. It has been shown that for specific MHD configurations a positive local magnetic shear in a region of unfavorable normal magnetic curvature yields higher linear growth rates. Estimates of the perpendicular diffusion coefficient based on mixing length theory and Fokker-Planck theory have also been computed and compared. 


\section{APPENDIX A: REMARKS ON THE LOCAL MAGNETIC SHEAR}

Using the definitions of the unit parallel vector, $\widehat{\mathbf{b}}=\mathbf{B} / B$, and the unit normal vector, $\widehat{\mathbf{n}}=\boldsymbol{\nabla} \psi / \sqrt{g^{\psi \psi}}$, we can write the binormal vector as

$$
\widehat{\mathbf{g}}=f(\mathbf{B} \times \nabla \psi),
$$

where $f=\left(B \sqrt{g^{\psi \psi}}\right)^{-1}$. Using Eq.(A1) in the definition of the local magnetic shear we obtain

$$
\begin{aligned}
S & =\widehat{\mathrm{g}} \cdot \boldsymbol{\nabla} \times \widehat{\mathrm{g}}=\widehat{\mathrm{g}} \cdot[\boldsymbol{\nabla} f \times(\mathbf{B} \times \boldsymbol{\nabla} \psi)+\boldsymbol{\nabla} \times(\mathbf{B} \times \boldsymbol{\nabla} \psi)], \\
& =f(\boldsymbol{\nabla} g^{\psi \psi} \cdot \underbrace{\nabla \alpha \times \widehat{\mathrm{g}}}_{\mathbf{1}}-\boldsymbol{\nabla} g^{\alpha \psi} \underbrace{\boldsymbol{\nabla} \psi \times \widehat{\mathrm{g}}}_{\mathbf{2}}),
\end{aligned}
$$

where we have used the Clebsch for the magnetic field, $\mathbf{B}=\boldsymbol{\nabla} \alpha \times \boldsymbol{\nabla} \psi$. Terms $\mathbf{1}$ and $\mathbf{2}$ in Eq.(A2) can be written as

$$
\boldsymbol{\nabla} \alpha \times \widehat{\mathrm{g}}=g \boldsymbol{\nabla} \alpha \times(\mathbf{B} \times \boldsymbol{\nabla} \psi)=f g^{\alpha \psi} \mathbf{B}
$$

and

$$
\boldsymbol{\nabla} \psi \times \widehat{\mathbf{g}}=f \boldsymbol{\nabla} \psi \times(\mathbf{B} \times \nabla \psi)=\frac{\sqrt{g^{\psi \psi}}}{B} \mathbf{B},
$$

respectively. Combining Eqs.(A2-A4), one can write

$$
\begin{aligned}
S & =f\left(f g^{\alpha \psi} \mathbf{B} \cdot \boldsymbol{\nabla} g^{\psi \psi}-\frac{\sqrt{g^{\psi \psi}}}{B} \mathbf{B} \cdot \boldsymbol{\nabla} g^{\alpha \psi}\right), \\
& =-\frac{|\boldsymbol{\nabla} \psi|^{2}}{B^{2}} \mathbf{B} \cdot \boldsymbol{\nabla}\left(\frac{g^{\alpha \psi}}{g^{\psi \psi}}\right), \\
& =\frac{|\boldsymbol{\nabla} \psi|^{2}}{B^{2}} \mathbf{B} \cdot \boldsymbol{\nabla}\left(D+\zeta \frac{d \iota}{d \psi}\right),
\end{aligned}
$$

where

$$
D \equiv \frac{\iota \boldsymbol{\nabla} \zeta \cdot \boldsymbol{\nabla} \psi-\boldsymbol{\nabla} \theta \cdot \boldsymbol{\nabla} \psi}{\boldsymbol{\nabla} \psi}
$$




\section{APPENDIX B: JACOBIAN FOR THE HELICAL PARAMETERIZATION}

In this Appendix, we consider the case of a configuration with a helical axis

$$
\begin{aligned}
R & =R_{0}+\rho \cos \theta+\Delta \cos \left(N_{p} \zeta\right) \\
\phi & =-\zeta \\
Z & =\rho \sin \theta+\Delta \sin \left(N_{p} \zeta\right)
\end{aligned}
$$

where $\Delta$ is a measure of the helical excursion of the magnetic axis. Using the helical parameterization (B1), we obtain the following covariant metric elements

$$
\begin{aligned}
& g_{\theta \theta}=\rho^{2}, \\
& g_{\theta \zeta}=N_{p} \rho \Delta \cos \left(N_{p} \zeta-\theta\right), \\
& g_{\zeta \zeta}=R^{2}+N_{p}^{2} \Delta^{2} .
\end{aligned}
$$

The Jacobian constraint equation is given by

$$
\frac{\partial}{\partial \theta}\left(\frac{F}{\partial}\right)=\frac{\partial}{\partial \zeta}\left(\frac{G}{\partial}\right)
$$

where $F(\theta, \zeta)=g_{\zeta \zeta}+\iota g_{\theta \zeta}=R^{2}+N_{p}^{2} \Delta^{2}+N_{p} \rho \Delta \iota \cos \varphi$ and $G(\theta, \zeta)=g_{\theta \zeta}+\iota g_{\theta \theta}=\iota \rho^{2}+$ $N_{p} \rho \Delta \cos \varphi$, where $\varphi \equiv N_{p} \zeta-\theta$ is the helical coordinate. Using the change of variable $(\theta, \zeta) \mapsto(\varphi, \bar{\theta})$ with

$$
\begin{aligned}
& \varphi=N_{p} \zeta-\theta, \\
& \bar{\theta}=\theta,
\end{aligned}
$$

in Eq.(B3), we arrive at

$$
\frac{\partial}{\partial \theta}\left(\frac{F}{\partial}\right)=\frac{\partial}{\partial \varphi}\left(\frac{N_{p} G+F}{\mathcal{J}}\right),
$$

where $\bar{\theta} \equiv \theta$. Let $X(\theta)=R_{0}+\rho \cos \theta$. For $\Delta=0$, we have the relations of $F=X^{2}(\theta)$ and $G=\iota \rho^{2}$. The Jacobian constraint admits the exact solution of $\mathcal{J}=C X^{2}(\theta)$, where $C$ is a constant. Let us seek a solution of the form

$$
\mathcal{J}=\frac{X^{2}(\theta)}{A(\varphi)}
$$

Substituting the trial function (B6) in Eq.(B5) we obtain

$$
\left(N_{p} G+F\right) \frac{d A}{d \varphi}=f(\theta, \zeta) A(\varphi)
$$


where

$$
f(\theta, \zeta)=2 \rho \sin \theta\left(\frac{F}{X}-R\right)+N_{p}\left(N_{p}+\iota\right) \rho \Delta \sin \varphi
$$

We can write $R=R_{0}\left[1+\epsilon_{\mathrm{t}} \cos \theta+\epsilon_{\mathrm{h}} \cos \left(N_{p} \zeta\right)\right]$ where $\epsilon_{\mathrm{t}} \equiv \rho / R_{0}$ and $\epsilon_{\mathrm{h}} \equiv \Delta / R_{0}$ are termed the toroidal parameter anf the helical parameter, respectively. After some algebra, we obtain the relation of

$$
\begin{aligned}
\frac{F}{X}-R & \simeq R_{0}\left\{\epsilon_{\mathrm{h}} \cos (\varphi+\theta)+\left(N_{p}^{2} \epsilon_{\mathrm{h}}^{2}+N_{p} \epsilon_{\mathrm{t}} \epsilon_{\mathrm{h}} \iota \cos \varphi\right)\left(1-\epsilon_{\mathrm{t}} \cos \theta\right)\right. \\
& \left.+\epsilon_{\mathrm{h}}{ }^{2} \cos ^{2}(\varphi+\theta)\left(1-\epsilon_{\mathrm{t}} \cos \theta\right)\right\}
\end{aligned}
$$

Introducing the operator

$$
\bar{F} \equiv \frac{1}{2 \pi} \int_{0}^{2 \pi} F(\theta) d \theta
$$

and noting the relations of

$$
\begin{aligned}
\frac{\cos (\varphi+\theta) \sin \theta}{\frac{\sin \varphi}{2},} & =-\frac{\cos ^{2}(\varphi+\theta) \sin \theta}{\cos ^{2}}=0, \\
\frac{\sin \theta \cos ^{2}(\varphi+\theta)}{\cos } & =-\frac{1}{4} \cos \varphi \sin \varphi,
\end{aligned}
$$

one can show that

$$
\bar{f}=-R_{0} \rho \epsilon_{\mathrm{h}} \sin \varphi\left(1-\frac{\widehat{\epsilon}}{2} \cos \varphi\right)+N_{p}\left(N_{p}+\iota\right) \rho \Delta \sin \varphi,
$$

where $\widehat{\epsilon} \equiv \epsilon_{\mathrm{t}} \epsilon_{\mathrm{h}}$. Similarly one has

$$
\begin{gathered}
\overline{R^{2}}=R_{0}^{2}\left(1+\frac{\epsilon_{\mathrm{t}}{ }^{2}}{2}+\widehat{\epsilon} \cos \varphi+\frac{\epsilon_{\mathrm{h}}^{2}}{2}\right), \\
\bar{F}=R_{0}^{2}\left[1+\frac{\epsilon_{\mathrm{t}}{ }^{2}}{2}+\left(N_{p}^{2}+\frac{1}{2}\right) \epsilon_{\mathrm{h}}{ }^{2}+\widehat{\epsilon}\left(1+N_{p} \iota\right) \cos \varphi\right],
\end{gathered}
$$

and

$$
\bar{G}=R_{0}^{2}\left(N_{p} \widehat{\epsilon} \cos \varphi+\iota \epsilon_{\mathrm{t}}^{2}\right)
$$

Using Eqs.(B11-B14), we obtain the relation of

$$
(\widehat{\alpha}+\widehat{\beta} \cos \varphi) \frac{d A}{d \varphi}=\xi \sin \varphi A
$$


where $\widehat{\alpha}=1+\epsilon_{\mathrm{t}}{ }^{2}\left(N_{p} \iota+\frac{1}{2}\right)+\epsilon_{\mathrm{h}}{ }^{2}\left(N_{p}^{2}+\frac{1}{2}\right), \widehat{\beta}=\widehat{\epsilon}\left[1+N_{p}\left(N_{p}+\iota\right)\right]$ and $\xi=$ $\left[N_{p}\left(N_{p}+\iota\right)-1\right] \widehat{\epsilon}$. Eq.(B15) can be solved perturbatively in ascending order of the smallness parameter $\epsilon \equiv N_{p}^{2} \widehat{\epsilon}$ by writing

$$
A=A_{0}+A_{1} \epsilon+A_{2} \epsilon^{2}+\cdots
$$

We have the relations of

$$
\begin{gathered}
\widehat{\alpha} \frac{d A_{0}}{d \varphi}=0 \quad\left[\mathcal{O}\left(\epsilon^{0}\right)\right], \\
\widehat{\beta} \cos \varphi \frac{d A_{0}}{d \varphi}+\widehat{\alpha} \frac{d A_{1}}{d \varphi}=A_{0} \xi \sin \varphi \quad[\mathcal{O}(\epsilon)],
\end{gathered}
$$

and

$$
\widehat{\beta} \frac{d A_{2}}{d \varphi}+\widehat{\beta} \cos \varphi \frac{d A_{1}}{d \varphi}=A_{1} \xi \sin \varphi \quad\left[\mathcal{O}\left(\epsilon^{2}\right)\right]
$$

Eqs.(B17-B19) can be solved with the result of

$$
\begin{gathered}
A_{0}=\text { const } \\
A_{1}=A_{0} \frac{\xi}{\widehat{\alpha}}(1-\cos \varphi),
\end{gathered}
$$

and

$$
A_{2}=A_{0} \frac{\xi}{\widehat{\alpha}^{2}}\left[\xi(1-\cos \varphi)-\frac{\widehat{\beta}+\xi}{2} \sin ^{2} \varphi\right] .
$$

It follows that the Jacobian can be written as

$$
\mathcal{J}=\frac{X^{2}(\theta)}{A_{0}(\psi) g(\varphi)}
$$

where $g(\varphi)=1+a(1-\cos \varphi)-b \sin ^{2} \varphi, a=\xi(1+\xi / \widehat{\alpha}) / \widehat{\alpha}$ and $b=\xi(\widehat{\beta}+\xi) /\left(2 \widehat{\alpha}^{2}\right)$. 
Acknowledgments This research was supported by Contract No. DE-AC02-CH0-3073 and the Scientific Discovery through Advanced Computing (SciDAC) initiative (U.S. Department of Energy). 
[1] P. C. Liewer, Nucl. Fusion 25, 543 (1985).

[2] W. Horton, Rev. Mod. Phys. 71, 735 (1999).

[3] W. Horton, Phys. Fluids B1(3), 524 (1989).

[4] A. Bhattacharjee, J. E. Sedlak, P. L. Similon, M. N. Rosenbluth and D. W. Ross, Phys. Fluids 26(4), 880 (1983).

[5] M. Persson, J. L. V. Lewandowski and H. Nordman, Phys. Plasmas 2(9), 3440 (1996).

[6] A. Zagorodny and J. Weiland, Phys. Plasmas 6(6), 2359 (1999).

[7] N. Dominguez, B. A. Carreras and V. E. Lynch, Phys. Fluids B4 (9), 2894 (1992).

[8] T. M. Antonsen and J. B. Lane, Phys. Fluids 23(6), 1205 (1980).

[9] L. I. Rudakov and R. Z. Sagdeev, Sov. Phys. Dokl. 6(5), 415 (1961).

[10] O. P. Pogutse, Sov. Phys. JETP 25, 498 (1967).

[11] B. Coppi, M. N. Rosenbluth and R. Z. Sagdeev, Phys. Fluids 10, 582 (1967).

[12] A. B. Hassam, T. M. Antonsen, J. F. Drake and P. N. Gudzar, Phys. Fluids B2 (8), 1822 (1990).

[13] W. Horton, D. Choi and W. M. Tang, Phys. Fluids 24(8), 1077 (1981).

[14] B. B. Kadomtsev and O. P. Pogutse, Nucl. Fusion 11, 67 (1971).

[15] P. H. Rutherford and E. A. Frieman, Phys. Fluids 11(3), 569 (1968).

[16] I.S. Gradshteyn and I.M. Ryzhik, Table of Integrals, Series, and Products, (Academic Press, New York, 1965).

[17] S.C. Guo and J. Weiland, Nucl. Fusion 37(8), 1095 (1997).

[18] P.N. Guzdar, L. Chen, W. Tang and P.H. Rutherford, Phys. Fluids 26(3), 673 (1983).

[19] J.W. Connor, R.J. Hastie and J.B. Taylor, Proc. Roy. Soc. London A 365, 1 (1979).

[20] C.C. Hegna, Phys. Plasmas 7(10), 3921 (2000).

[21] J.L.V. Lewandowski, Drift wave models for three-dimensional plasmas (Ph.D. Thesis, the Australian National University, 1997).

[22] M. Nadeem, T. Rafiq and M. Persson, Phys. Plasmas 8(10), 4375 (2001). 
Figure 1 Schematic representation of the cross section $\zeta=0$ (see main text for definition of the average minor radius, $\bar{a})$.

Figure 2 Linear growth rate as a function of the ion temperature gradient parameter, $\eta_{i}$ for the initial-value code (plain line) and the eigenmode analysis (dotted line)

Figure 3 Linear growth rate measured using 2 different methods as a function of time.

Figure 4 Mode frequency measured using 2 different methods as a function of time.

Figure 5 Real (plain line) and imaginary (dotted line) parts of the most stable eigenfunction of the electrostatic potential.

Figure 6 Dependence of the saturated linear growth rate as a function of the parameter $\bar{\theta}_{\text {max }} ; \gamma_{\infty}$ denotes the linear growth rate for $\bar{\theta}_{\text {max }} \mapsto \infty$.

Figure 7 Saturated linear growth rate as a function of the parameter $V_{\perp \max }$.

Figure 8 Real mode frequency as a function of the parameter $k_{\theta} \rho_{\mathrm{s}}$.

Figure 9 Linear growth rate as a function of the parameter $k_{\theta} \rho_{\mathrm{s}}$.

Figure 10 Mode frequency as a function of the helical parameter $\epsilon_{\mathrm{h}}$.

Figure 11 Linear growth rate as a function of the helical parameter $\epsilon_{\mathrm{h}}$.

Figure 12 Normal curvature (plain line) and local magnetic shear (dotted line) for the set $\left(\epsilon_{\mathrm{t}}, \epsilon_{\mathrm{h}}\right)=(0.0,0.1)$.

Figure 13 Normal curvature (plain line) and local magnetic shear (dotted line) for the set $\left(\epsilon_{\mathrm{t}}, \epsilon_{\mathrm{h}}\right)=(0.0,0.12)$.

Figure 14 Perpendicular diffusion coefficients $D_{\perp}^{(m)}$ and $D_{\perp}^{(f p)}$ as a function of the helical parameter $\epsilon_{\mathrm{h}}$. 
Figure 1 Lewandowski

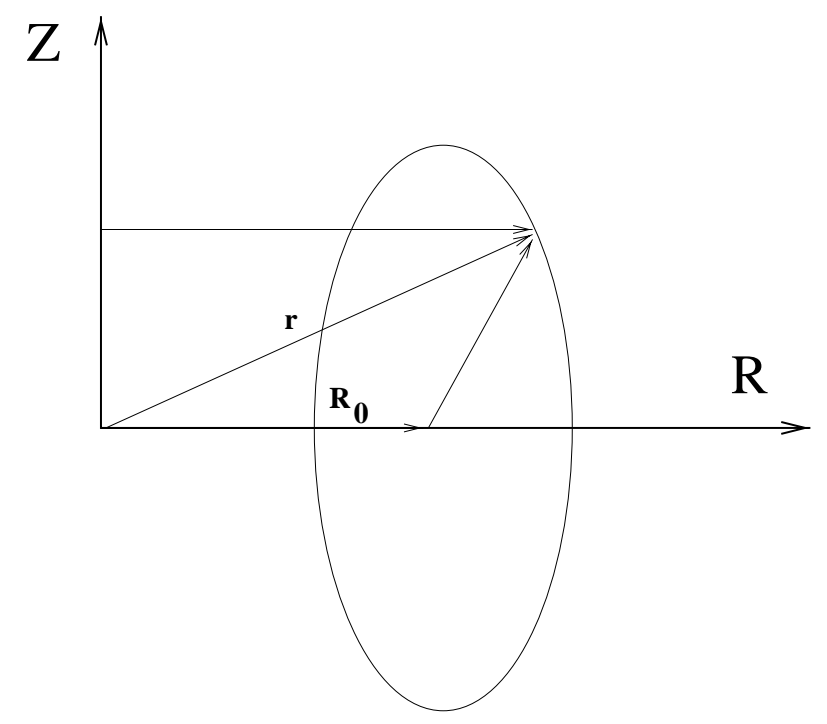


Figure 2 Lewandowski

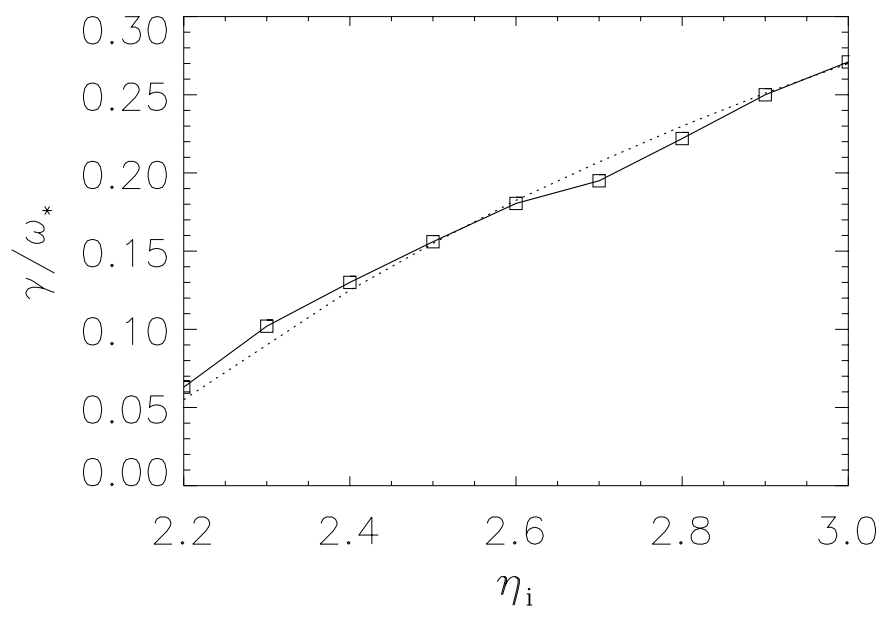


Figure 3 Lewandowski

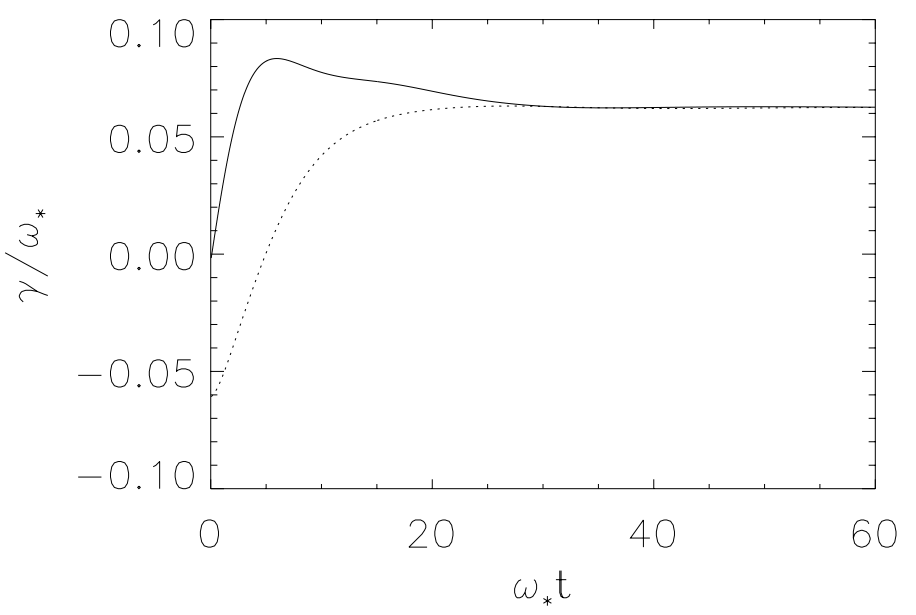


Figure 4 Lewandowski

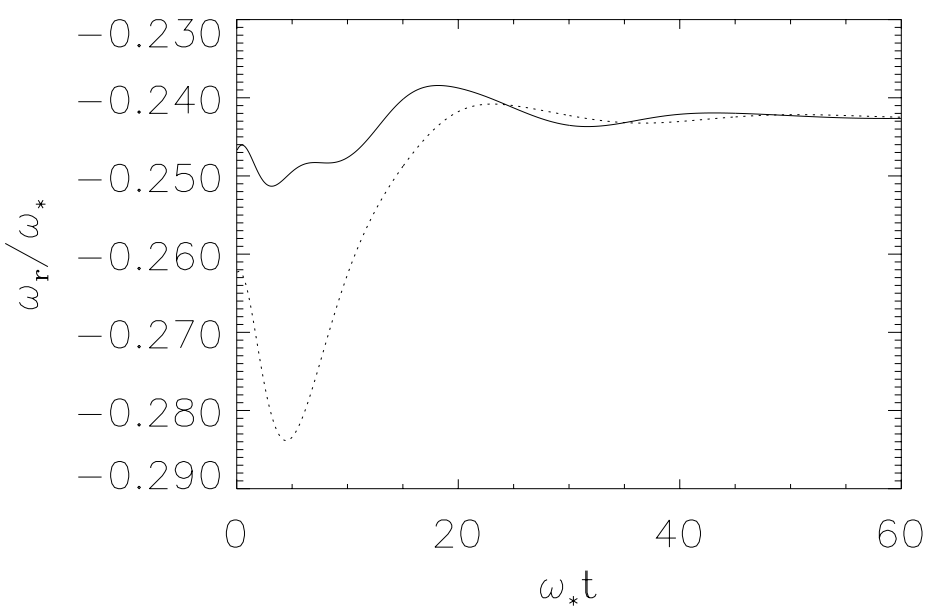


Figure 5 Lewandowski

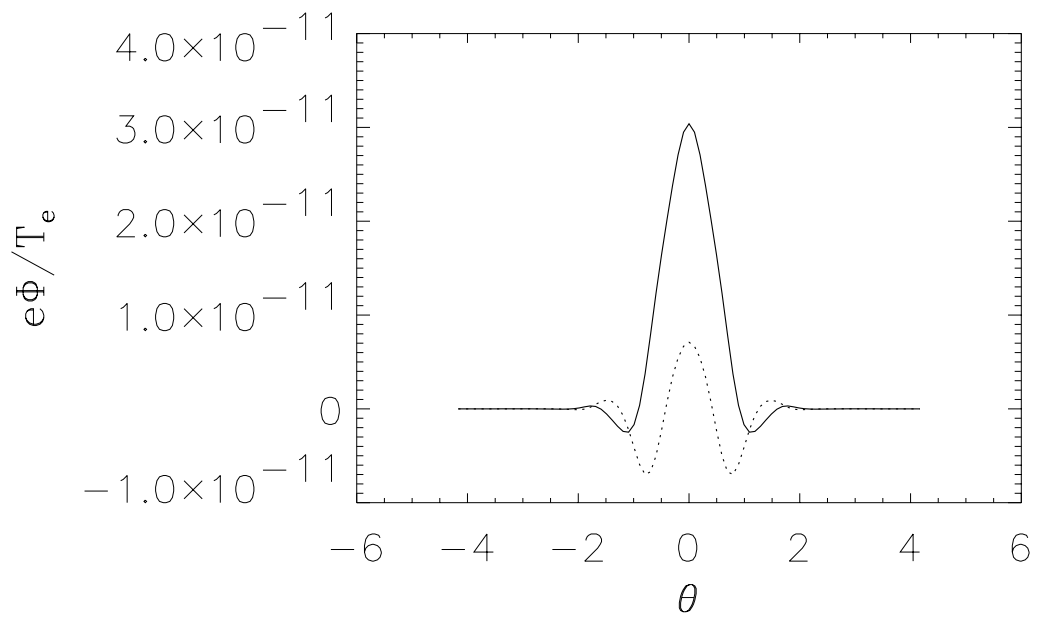


Figure 6 Lewandowski

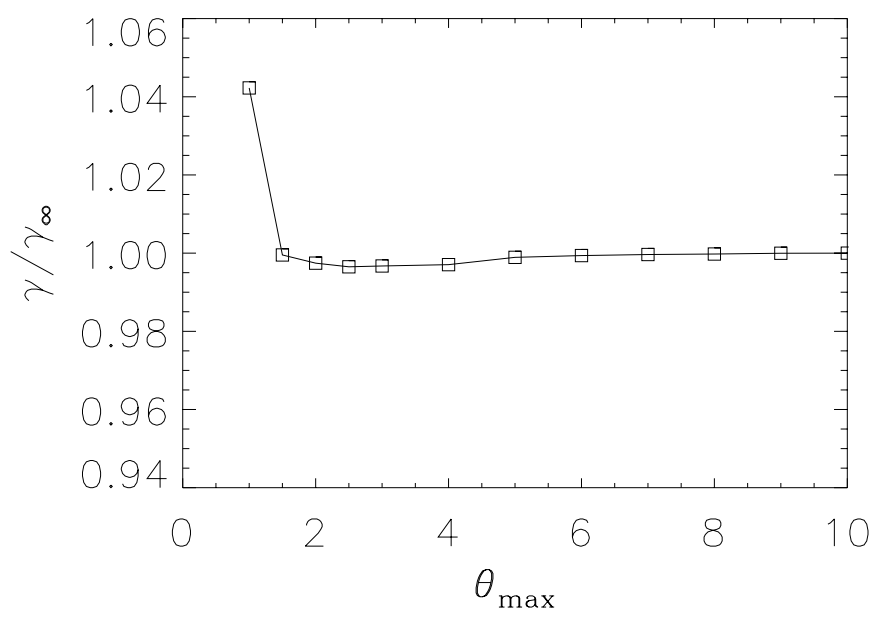


Figure 7 Lewandowski

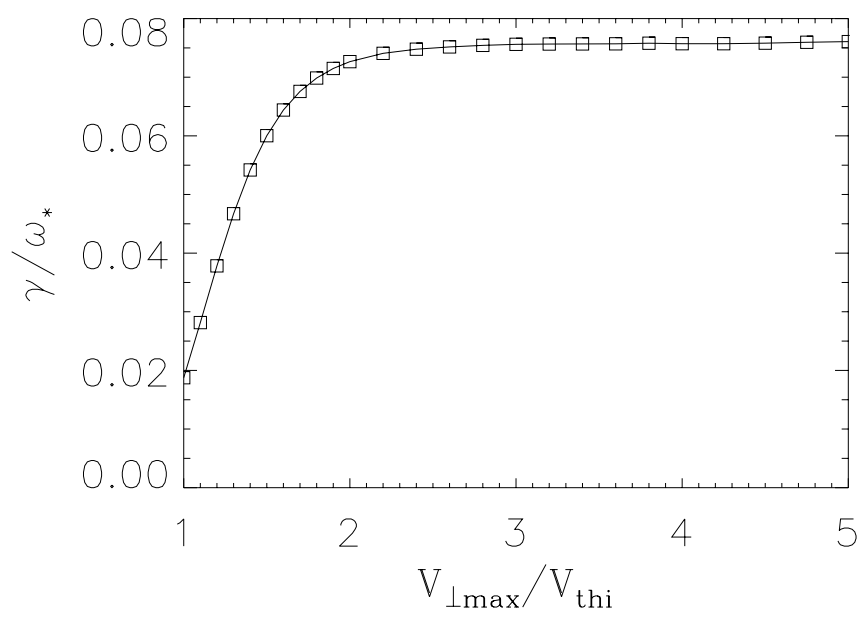


Figure 8 Lewandowski

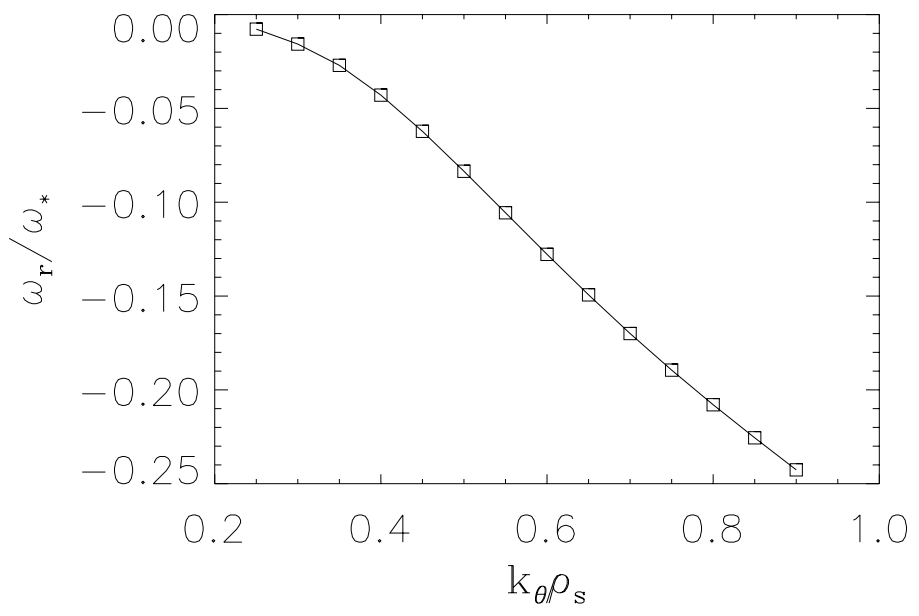


Figure 9 Lewandowski

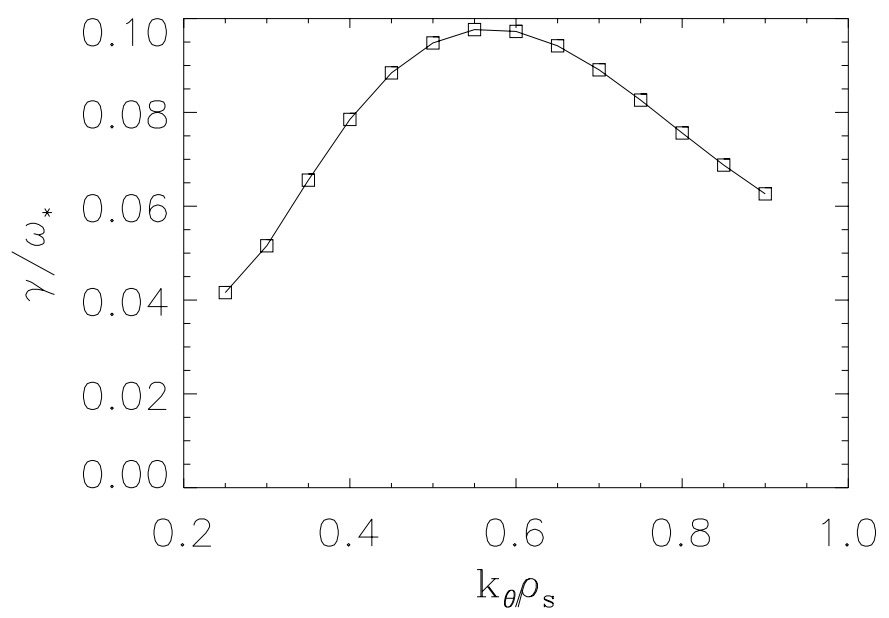




\section{Figure 10 Lewandowski}

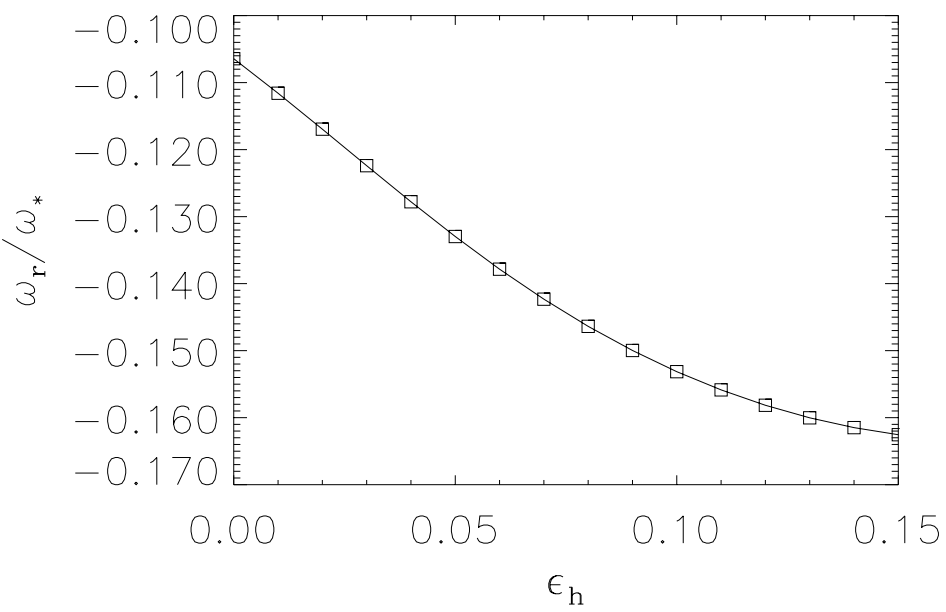




\section{Figure 11 Lewandowski}

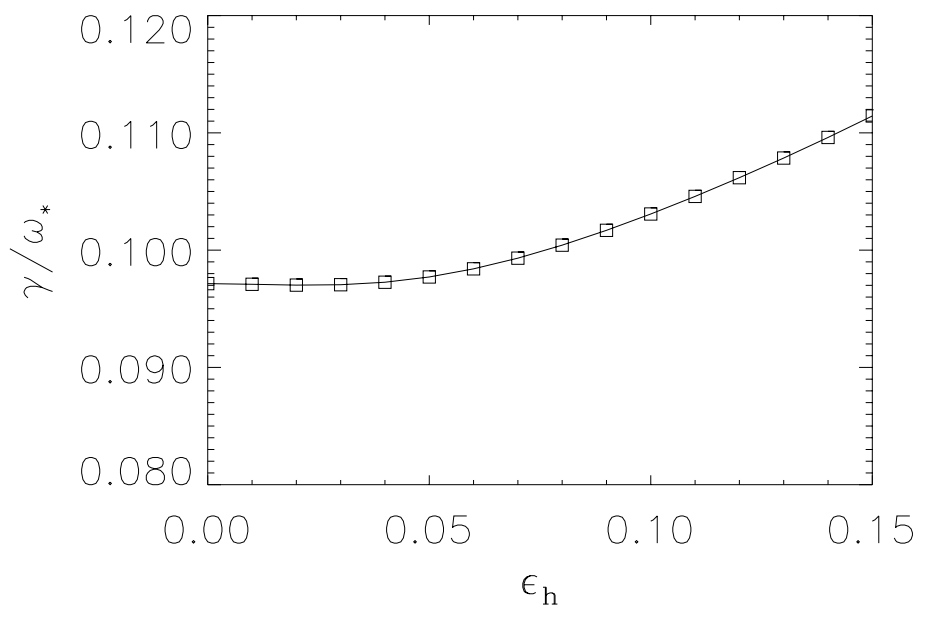


Figure 12 Lewandowski

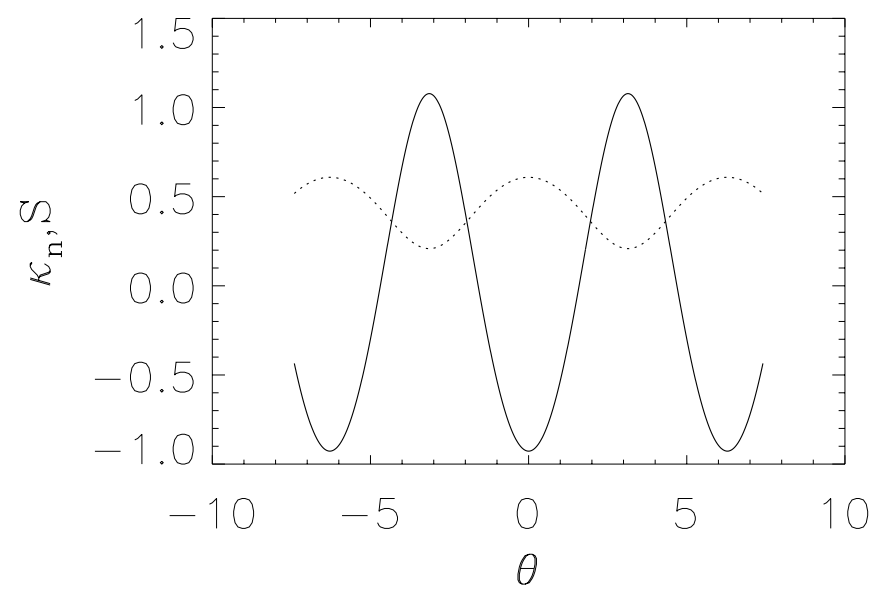


Figure 13 Lewandowski

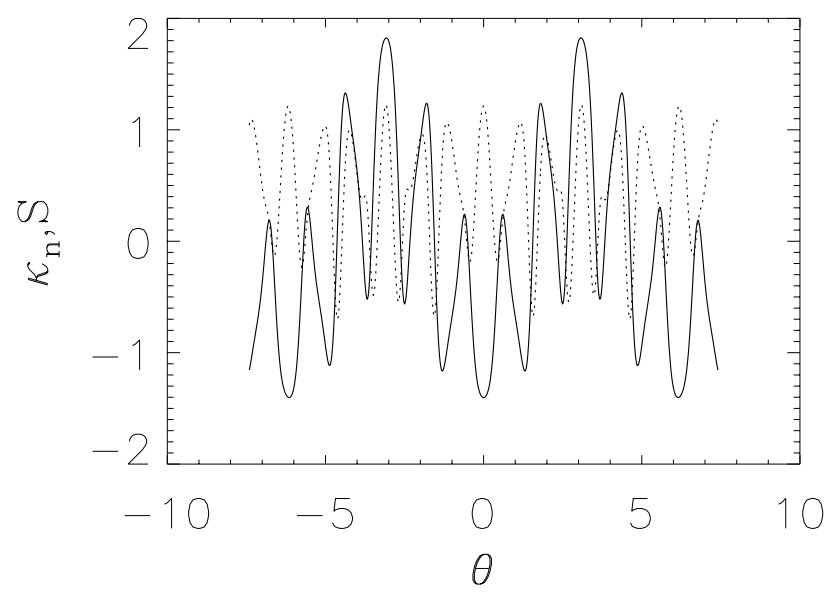




\section{Figure 14 Lewandowski}

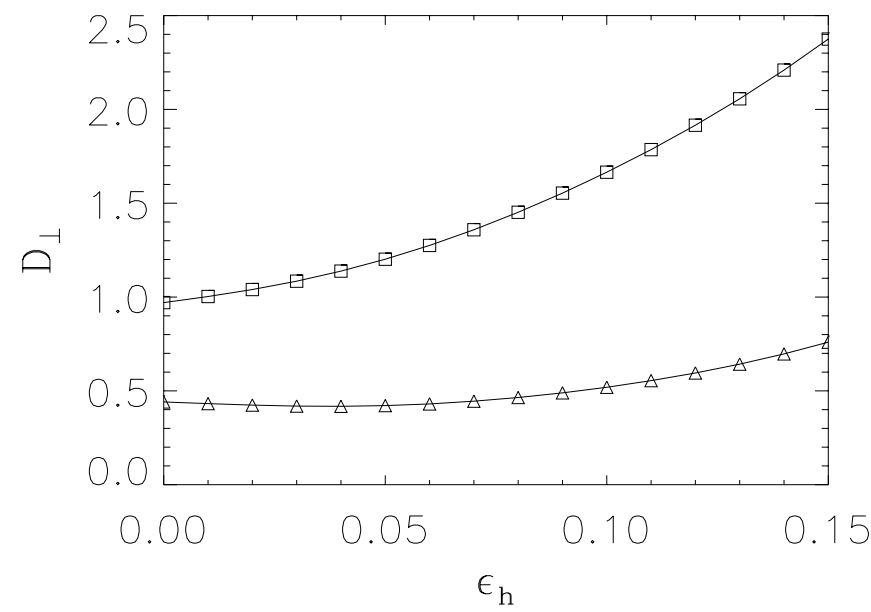




\section{External Distribution}

Plasma Research Laboratory, Australian National University, Australia

Professor I.R. Jones, Flinders University, Australia

Professor João Canalle, Instituto de Fisica DEQ/IF - UERJ, Brazil

Mr. Gerson O. Ludwig, Instituto Nacional de Pesquisas, Brazil

Dr. P.H. Sakanaka, Instituto Fisica, Brazil

The Librarian, Culham Laboratory, England

Mrs. S.A. Hutchinson, JET Library, England

Professor M.N. Bussac, Ecole Polytechnique, France

Librarian, Max-Planck-Institut für Plasmaphysik, Germany

Jolan Moldvai, Reports Library, Hungarian Academy of Sciences, Central Research Institute for Physics, Hungary

Dr. P. Kaw, Institute for Plasma Research, India

Ms. P.J. Pathak, Librarian, Institute for Plasma Research, India

Ms. Clelia De Palo, Associazione EURATOM-ENEA, Italy

Dr. G. Grosso, Instituto di Fisica del Plasma, Italy

Librarian, Naka Fusion Research Establishment, JAERI, Japan

Library, Laboratory for Complex Energy Processes, Institute for Advanced Study, Kyoto University, Japan

Research Information Center, National Institute for Fusion Science, Japan

Dr. O. Mitarai, Kyushu Tokai University, Japan

Dr. Jiangang Li, Institute of Plasma Physics, Chinese Academy of Sciences, People's Republic of China

Professor Yuping Huo, School of Physical Science and Technology, People's Republic of China

Library, Academia Sinica, Institute of Plasma Physics, People's Republic of China

Librarian, Institute of Physics, Chinese Academy of Sciences, People's Republic of China

Dr. S. Mirnov, TRINITI, Troitsk, Russian Federation, Russia

Dr. V.S. Strelkov, Kurchatov Institute, Russian Federation, Russia

Professor Peter Lukac, Katedra Fyziky Plazmy MFF UK, Mlynska dolina F-2, Komenskeho Univerzita, SK-842 15 Bratislava, Slovakia

Dr. G.S. Lee, Korea Basic Science Institute, South Korea

Institute for Plasma Research, University of Maryland, USA

Librarian, Fusion Energy Division, Oak Ridge National Laboratory, USA

Librarian, Institute of Fusion Studies, University of Texas, USA

Librarian, Magnetic Fusion Program, Lawrence Livermore National Laboratory, USA

Library, General Atomics, USA

Plasma Physics Group, Fusion Energy Research Program, University of California at San Diego, USA

Plasma Physics Library, Columbia University, USA

Alkesh Punjabi, Center for Fusion Research and Training, Hampton University, USA

Dr. W.M. Stacey, Fusion Research Center, Georgia Institute of Technology, USA

Dr. John Willis, U.S. Department of Energy, Office of Fusion Energy Sciences, USA

Mr. Paul H. Wright, Indianapolis, Indiana, USA 
The Princeton Plasma Physics Laboratory is operated by Princeton University under contract with the U.S. Department of Energy.

\author{
Information Services \\ Princeton Plasma Physics Laboratory \\ P.O. Box 451 \\ Princeton, NJ 08543
}

Phone: 609-243-2750

Fax: 609-243-2751

e-mail: pppl_info@pppl.gov

Internet Address: http://www.pppl.gov 